\title{
Characterization of Bijective Discretized Rotations
}

Bertrand Nouvel, Eric Rémila

École Normale Supérieure de Lyon

46 Allée d'Italie, 69364 Lyon Cedex 07, France

Téléphone : $+33(0) 4.72 .72 .80 .37$

Télécopieur : +33(0)4.72.72.80.80

Adresse électronique : lip@ens-lyon.fr 


\title{
Characterization of Bijective Discretized Rotations
}

\author{
Bertrand Nouvel, Eric Rémila
}

\begin{abstract}
A discretized rotation is the composition of an Euclidean rotation with the rounding operation. For $0<\alpha<\pi / 4$, we prove that the discretized rotation $\left[r_{\alpha}\right]$ is bijective if and only if there exists a positive integer $k$ such as
\end{abstract}

$$
\{\cos \alpha, \sin \alpha\}=\left\{\frac{2 k+1}{2 k^{2}+2 k+1}, \frac{2 k^{2}+2 k}{2 k^{2}+2 k+1}\right\}
$$

The proof uses a particular subgroup of the torus $(\mathbb{R} / \mathbb{Z})^{2}$.

Keywords: discrete rotations, pythagorean triple, integer pythagorean triples, bijective rotations, local description

\section{Résumé}

La rotation discrétisée est la composition d'une rotation euclidienne avec l'opération d'arrondi. Pour $0 \leq \alpha \leq \pi / 2$, nous montrons que la rotation discrétisée est bijective si et seulement il existe entier $k$ tel que les deux ensembles suivants soit identiques

$$
\{\cos \alpha, \sin \alpha\}=\left\{\frac{2 k+1}{2 k^{2}+2 k+1}, \frac{2 k^{2}+2 k}{2 k^{2}+2 k+1}\right\}
$$

La preuve exploite un sous groupe particulier du tore $(\mathbb{R} / \mathbb{Z})^{2}$.

Mots-clés: rotations discrètes, triples pythagoriciens, triples pythagoriciens entiers, rotations bijectives, description locale 


\section{Introduction}

In computer graphics, or in physical modeling, most of the time when a rotation has to be done, programmers simply use a discretized rotation, i. e. the composition of a classical rotation with a rounding operation. Unfortunately, this discrete rotation often has regrettable properties in terms of discrete geometry. Actually, for most angles the discretized rotation (restricted to $\mathbb{Z}^{2}$ ) is not bijective.

Nevertheless, in [5], Marie-Andrée Jacob and Eric Andrès have proved that for a certain subset of angles (the integer pythagorean angles), the discretized rotation is bijective. The proof relies on the classical formalism of discrete geometry, and a particular notion of underlying tile. But the question of the reciprocal was not mentioned and was left open - we did not know if there were some other bijective angles for discretized rotation.

In this paper, we exhibit an alternative proof to the Andres-Jacob result and we prove that the reciprocal is actually true : therefore we obtain a very simple characterization of the bijective angles for the discretized rotation.

In this article, we are going to start out with the minimal definitions we require; particularly those of the angles that are concerned by the Andrès-Jacob theorem. We continue by giving a characterization of surjective rotations : a discretized rotation is surjective if and only if no integer point has an image by Euclidean rotation and canonical projection to the torus $\mathbb{T}^{2}=(\mathbb{R} / \mathbb{Z})^{2}$ that stands inside a certain frame of the torus $\mathbb{T}^{2}$. The equivalence in between surjectivity and injectivity for the discretized rotation is then proved.

Afterward, to characterize angles that are bijective, we have examined a particular subgroup of the torus $\mathbb{T}^{2}$. Naturally, it is then described with great accuracy : more precisely we show that it is possible to identify the smallest vector. This vector can generate the whole studied group.

At the end, all these elements put back together allow us to reprove the Andrès-Jacob theorem and to prove its reciprocal.

\section{Pythagorean Angles and Triples}

An angle is a real number of the interval $[0 \ldots 2 \pi[$. For sake of simplicity, we will only study (without loss of generality by symmetry arguments) angles which belong to ]0, $\frac{\pi}{2}[$.

Definition 1 An angle $\alpha$ is pythagorean if $\cos \alpha$ and $\sin \alpha$ are both rational.

Notice that $\alpha$ is pythagorean if and only if $\alpha^{\prime}=\frac{\pi}{2}-\alpha$ is pythagorean.

Proposition 1 An angle $\alpha$ is pythagorean if and only if there exists a vector $\mathbf{v}$ of $\mathbb{Z}^{2} \backslash\{(0,0)\}$ such that $r_{\alpha}(\mathbf{v})$ also belongs to $\mathbb{Z}^{2}$.

Proof: Let $\alpha$ be a pythagorean angle. There exists an integer $C$ such that $C \cos \alpha$ and $C \sin \alpha$ both are integers. This can be interpreted as saying that $r_{\alpha}(C, 0)$ is in $\mathbb{Z}^{2}$. Conversely, let $\mathbf{v}=(x, y)$ be an integer vector such that $r_{\alpha}(\mathbf{v})$ is also in $\mathbb{Z}^{2}$. We state $\left(x^{\prime}, y^{\prime}\right)=r_{\alpha}(\mathbf{v})$. We have $\cos \alpha=\frac{x x^{\prime}+y y^{\prime}}{x^{2}+y^{2}}$ and $\sin \alpha=\frac{x y^{\prime}-y x^{\prime}}{x^{2}+y^{2}}$. This proves that $\cos \alpha$ and $\sin \alpha$ both are rational.

Any pair $(p, q)$ of positive integers such that $q<p$ can generate two pythagorean angles $\alpha$ and $\alpha^{\prime}$, such that $\alpha+\alpha^{\prime}=\frac{\pi}{2}$ : the first angle transforms $(p, q)$ into $(q, p)$, and the second one transforms $(q, p)$ into $(-q, p)$.

All the pythagorean angles generated as above can be generated with pairs $(p, q)$ such that $\operatorname{gcd}(p, q)=1$ (since, for each positive integer $h$, the angles generated by the pair $(h p, h q)$ are the same as those generated by $(p, q))$ and $p-q$ is odd (otherwise the angles generated by the pairs $(p, q)$ are the same as those generated $\left.\left(\frac{p+q}{2}, \frac{p-q}{2}\right)\right)$. The proposition below claims that the above process generates all the pythagorean angles.

Proposition 2 An angle $\alpha$ of $] 0, \frac{\pi}{2}\left[\right.$ is pythagorean if and only if there exists a vector $(p, q)$ of $\mathbb{Z}^{2}$ such that $p>q>0, \operatorname{gcd}(p, q)=1, p-q$ is odd and either $r_{\alpha}(p, q)=(q, p)$ or $r_{\alpha}(q, p)=(-q, p)$. 
Proof: This is a consequence of classical results related to pythagorean triples (See for example [4] or [10]). For any triple $(a, b, c)$ of positive integers such that $a^{2}+b^{2}=c^{2}$ and $\operatorname{gcd}(a, b, c)=1$, there exists a unique pair $(p, q)$ of positive integers such that $c=p^{2}+q^{2}$ and $\{a, b\}=\left\{p^{2}-q^{2}, 2 p q\right\}$. Obviously, we necessarily have $: p>q, \operatorname{gcd}(p, q)=1$, and $p-q$ odd. The proposition is just an application of this result for $(a, b, c)$ such that $\cos \alpha=a / c$ and $\sin \alpha=b / c$, and $c$ minimal.

Definition 2 The subset of pythagorean angles consisting in angles generated by pairs $(k+1, k)$ of consecutive integers is called the set of integer pythagorean angles.

Notice that the pair $(k+1, k)$ leads to the following triple : $(a=2 k(k+1), b=2 k+1$, $c=2 k(k+1)+1)$.

\section{Rotation Multiplicities, Holes and Double Points}

The rounding function is defined so : for each element $x$ of $\mathbb{R},[x]=\left\lfloor x+\frac{1}{2}\right\rfloor$ (the function floor which is written $\lfloor x\rfloor$, designates the unique integer such that $\lfloor x\rfloor \leq x<\lfloor x\rfloor+1)$. On vectors, the discretization is applied component by component : for each vector $\mathbf{v}=(x, y)$ of $\mathbb{R}^{2}$, we have $[\mathbf{v}]=([x],[y])$. The set of vectors of the real plane that are discretized to a same vector is called a cell.

Given an angle $\alpha$, the discretized rotation $\left[r_{\alpha}\right]$ is defined on the set $\mathbb{Z}^{2}$ as the composition of the Euclidean rotation $r_{\alpha}$ and of the rounding function [.].

Let $\mathbf{i}_{\alpha}$ and $\mathbf{j}_{\alpha}$ be the vectors defined as the rotated images of the vectors of canonical base of the plane $: \mathbf{i}_{\alpha}=r_{\alpha}(\mathbf{i})=(\cos \alpha, \sin \alpha)$ and $\mathbf{j}_{\alpha}=r_{\alpha}(\mathbf{j})=(-\sin \alpha, \cos \alpha)$.

The multiplicity $M_{\alpha}(\mathbf{w})$ maps each vector $\mathbf{w}$ of $\mathbb{Z}^{2}$ to the number of its antecedents by discretized rotation (notice that $M_{\alpha}$ is a planar configuration which can be obtained by projection (more exactly by morphism - i. e. cell by cell) of the planar coloration introduced in [7]). Formally, the application $M_{\alpha}$ is defined by the following equation :

$$
M_{\alpha}(\mathbf{w})=C A R D\left(\left\{\mathbf{v} \in \mathbb{Z}^{2},\left[r_{\alpha}\right](\mathbf{v})=\mathbf{w}\right\}\right)
$$

Each set of three different points of the grid $r_{\alpha}\left(\mathbb{Z}^{2}\right)$ contains at least two points that are at a distance of at least $\sqrt{2}$, and the ends of a segment of length of at least $\sqrt{2}$ cannot be in the same cell, therefore there cannot be three different points inside the same cell, thus the multiplicity $M_{\alpha}$ of any vector will never exceed 2.

A hole is a vector characterized by the fact that $M_{\alpha}(\mathbf{w})=0$ : there is a hole in $M_{\alpha}$ if and only if $\left[r_{\alpha}\right]$ is not surjective. A double point is characterized by the fact that $M_{\alpha}(\mathbf{w})=2$ : there is a double point in $M_{\alpha}$ if and only if $\left[r_{\alpha}\right]$ is not injective. The discretized rotation $\left[r_{\alpha}\right]$ is bijective if and only if for each vector $\mathbf{w}$ of $\mathbb{Z}^{2}, M_{\alpha}(\mathbf{w})=1$.

Let $P_{k}$ be the set of vectors of $\mathbb{Z}^{2}$ such as $M(\mathbf{v})=k$. Normally, $P_{0}$ is empty if and only if $\left[r_{\alpha}\right]$ is surjective and $P_{2}$ is empty if and only if $\left[r_{\alpha}\right]$ is injective.

We identify the torus $\mathbb{T}^{2}=(\mathbb{R} / \mathbb{Z})^{2}$ with the cell $\left[-\frac{1}{2},+\frac{1}{2}\left[{ }^{2}\right.\right.$. A canonical projection from $\mathbb{R}^{2}$ to $\mathbb{T}^{2}$ is provided by the operator $\{\mathrm{x}\}=\mathrm{x}-[\mathrm{x}]$. A frame is the cartesian product of non-empty half-opened intervals $\left[a \ldots b\left[\right.\right.$ on the torus $\mathbb{T}^{2}=(\mathbb{R} / \mathbb{Z})^{2}$.

Theorem 1 Let $F_{0 \downarrow}$ denote the frame:

$$
F_{0 \downarrow}=\left[\frac{1}{2}-\sin \alpha,-\frac{1}{2}+\cos \alpha\left[\times\left[-\frac{1}{2},-\frac{3}{2}+\cos \alpha+\sin \alpha[\right.\right.\right.
$$

We have : $P_{0} \neq \emptyset$ if and only if there exists a vector $\mathbf{v}$ of $\mathbb{Z}^{2}$ such that $\left\{r_{\alpha}\right\}(\mathbf{v}) \in F_{0 \downarrow}$.

More precisely, with the notations above, we have $M_{\alpha}\left(\left[r_{\alpha}\right](\mathbf{v})-\mathbf{j}\right)=0$.

Proof:

First notice that necessarily, for any vector $\mathbf{w}^{\prime}$ of $\mathbb{Z}^{2}$, there exists a vector $\mathbf{v}$ of $\mathbb{Z}^{2}$ such that the distance between $r_{\alpha}(\mathbf{v})$ and $\mathbf{w}^{\prime}$ is at most $\sqrt{2} / 2$. Let $\mathbf{w}$ be a hole, $\mathcal{H}$ be the discretization cell 
associated and $\mathcal{D}_{\mathbf{w}}$ be the closed disk centered in $\mathbf{w}$ of radius $\sqrt{2} / 2$. From the remark above, the set $\mathcal{D}_{\mathbf{w}} \cap r_{\alpha}\left(\mathbb{Z}^{2}\right)$ is not empty.

On the other hand, since $\mathcal{H}$ is a hole, $r_{\alpha}\left(\mathbb{Z}^{2}\right)$ does not meet $\mathcal{H}$, and, therefore, $r_{\alpha}\left(\mathbb{Z}^{2}\right)$ does not meet $\mathcal{H}+\mathbf{t}$, for any vector $\mathbf{t}$ of $r_{\alpha}\left(\mathbb{Z}^{2}\right)$ (since $r_{\alpha}\left(\mathbb{Z}^{2}\right)+\mathbf{t}=r_{\alpha}\left(\mathbb{Z}^{2}\right)$ ). In particular, we have : $r_{\alpha}\left(\mathbb{Z}^{2}\right) \cap\left(\cup_{\mathbf{t} \in \mathcal{T}}(H+\mathbf{t})\right)=\emptyset$, where $\mathcal{T}$ denotes the set $\mathcal{T}=\left\{\mathbf{t} \in r_{\alpha}\left(\mathbb{Z}^{2}\right), \mathbf{t}=x \mathbf{i}_{\alpha}+y \mathbf{j}_{\alpha},-1 \leq x \leq\right.$ $1,-1 \leq y \leq 1\}$.

Thus $r_{\alpha}\left(\mathbb{Z}^{2}\right)$ necessarily meets $\mathcal{D}_{\mathbf{w}} \backslash \cup_{\mathbf{t} \in \mathcal{T}}(H+\mathbf{t})$. This set is contained into the union of four squares (see figure 1) : the square $F_{0 \downarrow}+\mathbf{w}+\mathbf{j}$ and its translated copies by vectors $-\mathbf{i}_{\alpha},-\mathbf{j}_{\alpha}$ and $-\mathbf{i}_{\alpha}-\mathbf{j}_{\alpha}$.

Obviously, the fact that one of these squares encounters $r_{\alpha}\left(\mathbb{Z}^{2}\right)$ implies that each of them meets $r_{\alpha}\left(\mathbb{Z}^{2}\right)$. This is especially true for $F_{0 \downarrow}+\mathbf{w}+\mathbf{j}$ thus there exists $\mathbf{v}$ of $\mathbb{Z}^{2}$ such that $\left\{r_{\alpha}\right\}(\mathbf{v}) \in F_{0 \downarrow}$.

Conversely, if there exists a vector $\mathbf{v}$ of $\mathbb{Z}^{2}$ such that $\left\{r_{\alpha}\right\}(\mathbf{v}) \in F_{0 \downarrow}$, then there exists a vector $\mathbf{w}$ of $\mathbb{Z}^{2}$ such that $F_{0 \downarrow}+\mathbf{w}+\mathbf{j}$ contains $r_{\alpha}(\mathbf{v})$. Thus $F_{0 \downarrow}+\mathbf{w}+\mathbf{j}$ and its translated copies by vectors $-\mathbf{i}_{\alpha},-\mathbf{j}_{\alpha}$ and $-\mathbf{i}_{\alpha}-\mathbf{j}_{\alpha}$ meet $r_{\alpha}\left(\mathbb{Z}^{2}\right)$. This yields that $\mathbf{w}$ is a hole since each vector of its discretization cell is at a distance lower than 1 from an element of $r_{\alpha}\left(\mathbb{Z}^{2}\right)$.

Theorem 2 Let $F_{2}$ denote the frame:

$$
F_{2}=\left[-\frac{1}{2}, \frac{1}{2}-\cos \alpha\left[\times\left[-\frac{1}{2}, \frac{1}{2}-\sin \alpha[\right.\right.\right.
$$

We have : $P_{2} \neq \emptyset$ if and only if there exists a vector $\mathbf{v}$ of $\mathbb{Z}^{2}$ such that $\left\{r_{\alpha}\right\}(\mathbf{v}) \in F_{2}$.

More precisely, with the notations above, we have $M_{\alpha}\left(\left[r_{\alpha}\right](\mathbf{v})\right)=2$.

Proof: Since $r_{\alpha}\left(\mathbb{Z}^{2}\right)$ is invariant by rotation of angle $\pi / 2, P_{2} \neq \emptyset$ if and only if there exists $\mathbf{v}$ such that $\left[r_{\alpha}\right](\mathbf{v})=\left[r_{\alpha}\right](\mathbf{v}+\mathbf{i})$. This condition trivially gives the result.

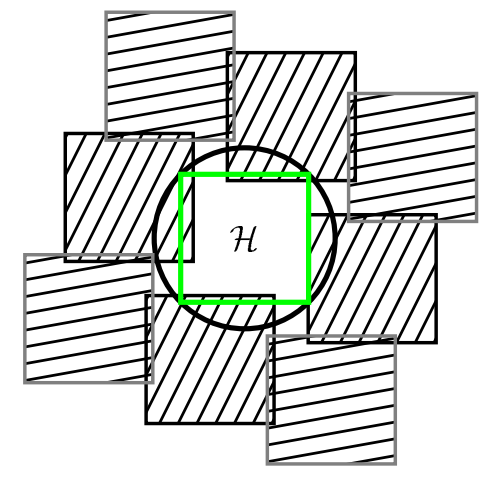

FIG. 1 - The main argument for Theorem 1 : The set $r_{\alpha}\left(\mathbb{Z}^{2}\right)$ meets neither the (big) dashed square nor $\mathcal{H}$, but meets the closed disk. Thus $r_{\alpha}\left(\mathbb{Z}^{2}\right)$ meets one of the four remaining small squares.

\subsection{The Non Pythagorean Case}

Now, we introduce two groups that have a main importance for the study of discrete rotations :

- the subgroup $\mathcal{G}_{\alpha}^{\prime}$ of $\mathbb{T}^{2}$ defined by $: \mathcal{G}_{\alpha}^{\prime}=\left\{r_{\alpha}\right\}\left(\mathbb{Z}^{2}\right)$ which one may also see as : $\mathbb{Z}\left\{\mathbf{i}_{\alpha}\right\}+\mathbb{Z}\left\{\mathbf{j}_{\alpha}\right\}$

- the subgroup $\mathcal{G}_{\alpha}$ of $\mathbb{R}^{2}$ defined by $: \mathcal{G}_{\alpha}=\mathbb{Z} \mathbf{i}+\mathbb{Z} \mathbf{j}+\mathbb{Z} \mathbf{i}_{\alpha}+\mathbb{Z} \mathbf{j}_{\alpha}$.

At first glance, we notice that a point $\mathbf{v}$ of $\mathbb{R}^{2}$ belongs to $\mathcal{G}_{\alpha}$ if and only if $\{\mathbf{v}\}$ belongs to $\mathcal{G}_{\alpha}^{\prime}$ and, moreover $\left[-1 / 2,1 / 2\left[{ }^{2} \cap \mathcal{G}_{\alpha}=\mathcal{G}_{\alpha}^{\prime}\right.\right.$. We also notice that $\mathcal{G}_{\alpha}$ and $\mathcal{G}_{\alpha}^{\prime}$ are both invariant by rotation of angle $\pi / 2$.

We now focus on non pythagorean angles in order to show that in this case, the discretized rotation will be neither injective, nor surjective. 
Proposition 3 Let $\alpha$ denote a non pythagorean angle; For all $\epsilon>0$, there exists a vector $\mathbf{e}_{\epsilon}$ of $\mathcal{G}_{\alpha}$ such that $0<\left\|\mathbf{e}_{\epsilon}\right\| \leq \epsilon$. Moreover, $\mathcal{G}_{\alpha}$ contains the group $\mathbb{Z} \mathbf{e}_{\epsilon}+\mathbb{Z} \mathbf{e}_{\epsilon}^{\prime}$, with $\mathbf{e}_{\epsilon}^{\prime}=r_{\pi / 2}\left(\mathbf{e}_{\epsilon}\right)$.

Proof: Since $\alpha$ is not pythagorean, the elements of the sequence $\left(\left\{n \mathbf{i}_{\alpha}\right\}\right)_{n \in \mathbb{N}}$ are pairwise disjoint. Since all of them are in the compact square $[-1 / 2,1 / 2]^{2}$, there exists a subsequence $\left(\left\{n_{k} \mathbf{i}_{\alpha}\right\}\right)_{k \in \mathbb{N}}$ which converges. Thus the sequence $\left(\left\{n_{k+1} \mathbf{i}_{\alpha}\right\}-\left\{n_{k} \mathbf{i}_{\alpha}\right\}\right)_{k \in \mathbb{N}}$ is a non ultimately constant sequence which converges to $(0,0)$. Thus, for all $\epsilon>0$, there exists an integer $k$ such that $0<\left\|\left\{n_{k+1} \mathbf{i}_{\alpha}\right\}-\left\{n_{k} \mathbf{i}_{\alpha}\right\}\right\| \leq \epsilon$. This element is in $\mathcal{G}_{\alpha}$, which gives the result (the second part of the proposition is trivial).

Corollary 1 Let $\alpha$ denote a non pythagorean angle. The associated discretized rotation $\left[r_{\alpha}\right]$ is neither injective nor surjective.

Proof: Let $F$ be a fixed frame. From the above proposition applied for $\epsilon$ sufficiently small, the group $\mathcal{G}_{\alpha}^{\prime}$ has a non empty intersection with $F$. In particular, this is true for the frame of surjectivity $F_{0 \downarrow}$ and the frame of injectivity $F_{2}$. This gives the result, according to Theorem 1 and Theorem 2.

\section{The Pythagorean Case}

We fix a pair $(p, q)$ of positive integers such that $p>q, \operatorname{gcd}(p, q)=1$ and $p-q$ is odd. Let $\alpha$ be the angle such that $\cos \alpha=a / c$ and $\sin \alpha=b / c$, with $a=p^{2}-q^{2}, b=2 p q$ and $c=p^{2}+q^{2}$. We also state : $\alpha^{\prime}=\frac{\pi}{2}-\alpha$, the angle defined by the other triple $\left(a^{\prime}=2 p q, b^{\prime}=p^{2}-q^{2}, c=p^{2}+q^{2}\right)$.

For each pair $(x, y)$ of $\mathbb{Z}^{2}$ we have $\left[r_{\alpha}\right](x, y)=\left(x^{\prime}, y^{\prime}\right)$ if and only if $\left[r_{\alpha^{\prime}}\right](y, x)=\left(y^{\prime}, x^{\prime}\right)$. Thus $\left[r_{\alpha}\right]$ is bijective (resp. injective/ surjective) if and only if $\left[r_{\alpha^{\prime}}\right]$ is (resp. injective/ surjective).

For the sake of simplicity, we now assume that $a$ is odd ( $a$ is the first element of the triple associated with the angle). There is no loss of generality.

\subsection{Reduction to surjectivity}

We now prove that, for pythagorean angles, the bijectivity problem is equal to the surjectivity problem.

Lemma 1 (square lemma) Let $S$ be a half-opened square of the plane such that the vectors induced by its edges have integer components. The number of integer vectors contained in $S$ is equal to the area of $S$.

Proof: (sketch) The idea (see Figure 2) is to divide the square into three parts, two triangles and another one, and afterward translate the triangles to obtain a polygon with integer sides, vertical or horizontal, which is the disjoint union of two half opened squares. The main arguments used are the facts below :

- the lemma above obviously holds for any half-opened square whose (integer) sides are vertical or horizontal.

- two domains of the plane which can be mutually obtained by integer translation contain the same number of integer vectors

A precise choice can be made for boundaries, in order to get half opened squares at the end.

Theorem 3 Let $\alpha$ denote a pythagorean angle. The function $\left[r_{\alpha}\right]$ is be one-to-one if and only if it is onto. Thus bijectivity is equivalent to injectivity or surjectivity.

Proof: We have $r_{\alpha}((a,-b))=(c, 0)$ and $r_{\alpha}((b, a))=(0, c)$. Thus, for each vector $\mathbf{v}$ of $\mathbb{Z}^{2}$, we have $\left[r_{\alpha}\right](\mathbf{v}+(a,-b))=\left[r_{\alpha}\right](\mathbf{v})+(c, 0)$ and $\left[r_{\alpha}\right](\mathbf{v}+(b, a))=\left[r_{\alpha}\right](\mathbf{v})+(0, c)$. This yields that for each vector $\mathbf{w}$ of $\mathbb{Z}^{2}$, we have $M(\mathbf{w}+(c, 0))=M(\mathbf{w}+(0, c))=M(\mathbf{w})$. In other words, the multiplicity is a periodic function.

Consider the real window $\left[-\frac{1}{2},-\frac{1}{2}+c\left[^{2}\right.\right.$. From the periodicity seen above, $\left[r_{\alpha}\right]$ is injective if and only if there exists no integer vector $\mathbf{w}$ in $\left[-\frac{1}{2},-\frac{1}{2}+c\left[\left[^{2}\right.\right.\right.$ such that $M(\mathbf{w}) \geq 2$. Similarly, $\left[r_{\alpha}\right]$ is surjective if and only if there exists no integer vector $\mathbf{w}$ in $\left[-\frac{1}{2},-\frac{1}{2}+c\left[^{2}\right.\right.$, such that $M(\mathbf{w})=0$. 
On the other hand, for each vector $\mathbf{v}$ of $\mathbb{Z}^{2},\left[r_{\alpha}\right](\mathbf{v})$ is element of $\left[-\frac{1}{2},-\frac{1}{2}+c\left[^{2}\right.\right.$ if and only if $\mathbf{v}$ is element of $r_{-\alpha}\left(\left[-\frac{1}{2},-\frac{1}{2}+c\left[^{2}\right)\right.\right.$. From the square lemma, the square $r_{-\alpha}\left(\left[-\frac{1}{2},-\frac{1}{2}+c\left[^{2}\right)\right.\right.$ contains $c^{2}$ integer vectors, as the square $\left[-\frac{1}{2},-\frac{1}{2}+c\left[^{2}\right.\right.$. Thus there exists an integer vector $\mathbf{w}$ in $\left[-\frac{1}{2},-\frac{1}{2}+c\left[^{2}\right.\right.$ such that $M(\mathbf{w})=0$ if and only if there exists an integer vector $\mathbf{w}$ in $\left[-\frac{1}{2},-\frac{1}{2}+c\left[^{2}\right.\right.$ such that $M(\mathbf{w}) \geq 2$. This achieves the proof.

Notice, that instead of the square lemma, a corollary of the famous Pick's Theorem may also be used ${ }^{1}$. (Even if the proof of the previous lemma can be a little bit wiser in terms it could require less constraints.)
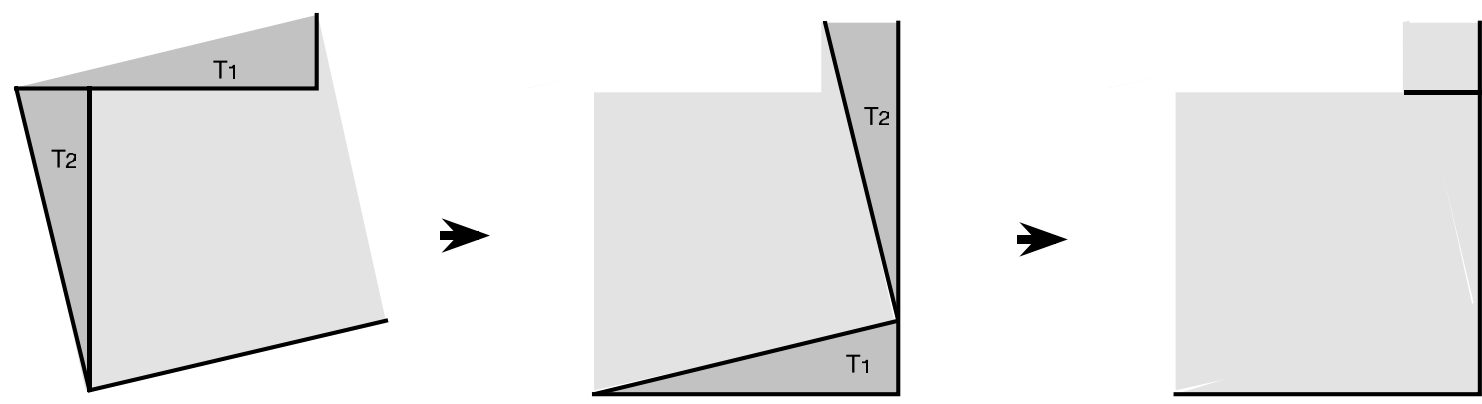

Fig. 2 - The scheme of the proof of the square lemma. The triangles $T_{1}$ and $T_{2}$ are translated, and two squares are obtained. The dark lines point out the boundaries which are inside the domains.

\subsection{Structural Study of $\mathcal{G}_{\alpha}^{\prime}$}

The Theorem 1 will be used to characterize surjective (i.e. bijective) rotations. But, it requires to know the precise structure of $\mathcal{G}_{\alpha}^{\prime}$ which is $\left\{r_{\alpha}\right\}\left(\mathbb{Z}^{2}\right)$

Lemma 2 (membership criterion) Let $\mathbf{v}=\left(\frac{x}{c}, \frac{y}{c}\right)$, with $(x, y)$ in $\mathbb{Z}^{2}$. There exists an integer $n$ such that $\left\{n \mathbf{i}_{\alpha}\right\}=\{\mathbf{v}\}$ if and only if $x b-y a=\operatorname{det}\left(\begin{array}{cc}x & a \\ y & b\end{array}\right) \equiv 0[c]$.

Proof: There exists an integer $n$ such that $\left\{n \mathbf{i}_{\alpha}\right\}=\{\mathbf{v}\}$ if and only if there exists a triple $\left(n, n^{\prime}, n^{\prime \prime}\right)$ of integers such that : $\left(\frac{x}{c}+n^{\prime}, \frac{y}{c}+n^{\prime \prime}\right)=n\left(\frac{a}{c}, \frac{b}{c}\right)$ (notice that $\left.\mathbf{i}_{\alpha}=\left(\frac{a}{c}, \frac{b}{c}\right)\right)$.

This is equivalent to the existence of an integer $n$ such that $x \equiv n a[c]$ and $y \equiv n b[c]$. And which is possible if and only if $x a^{-1} \equiv y b^{-1}[c]$ (the inverses are taken in $\mathbb{Z} / c \mathbb{Z}$, the numbers $a$ and $b$ both are invertible since $\frac{a}{c}$ and $\frac{b}{c}$ both are irreducible fractions). The latter equality can be rewritten : $x b-y a \equiv 0[c]$.

Proposition 4 Let $\mathbf{m}$ and $\mathbf{m}^{\prime}$ be the vectors defined by $\mathbf{m}=\left(\frac{p}{c}, \frac{q}{c}\right)$ and $\mathbf{m}^{\prime}=\left(\frac{-q}{c}, \frac{p}{c}\right)$. The group $\mathcal{G}_{\alpha}^{\prime}$ is cyclic, of order $c$, generated by the vector $\{\mathbf{m}\}$. The group $\mathcal{G}_{\alpha}$ is the subgroup of $\mathbb{R}^{2}$ generated by $\mathbb{Z} \mathbf{m}+\mathbb{Z} \mathbf{m}^{\prime}$.

Proof: We first notice that the set $\left\{\left\{\mathbf{i}_{\alpha}\right\},\left\{\mathbf{j}_{\alpha}\right\}\right\}$ generates $\mathcal{G}_{\alpha}^{\prime}$ and $\mathbf{j}_{\alpha}=\left(-\frac{b}{c}, \frac{a}{c}\right)$. Remark that $-b^{2}-a^{2}=-c^{2}$, thus, applying the membership criterion, there exists an integer $n$ such that $\left\{n \mathbf{i}_{\alpha}\right\}=\left\{\mathbf{j}_{\alpha}\right\}$. Thus $\left\{\mathbf{i}_{\alpha}\right\}$ generates $\mathcal{G}_{\alpha}^{\prime}$, which, therefore, is cyclic. Moreover, since $\operatorname{gcd}(a, c)=$ $\operatorname{gcd}(b, c)=1$, it stands that $:\left\{n \mathbf{i}_{\alpha}\right\}=(0,0)$ if and only if $n \equiv 0[c]$. It proves that the order of $\mathcal{G}_{\alpha}^{\prime}$ is $c$.

We have : $p b-q a=p(2 p q)-q\left(p^{2}-q^{2}\right)=p^{2} q+q^{3}=q\left(p^{2}+q^{2}\right)=q c$. Thus, applying the membership criterion, we obtain that $\{\mathbf{m}\}$ is an element of $\mathcal{G}_{\alpha}^{\prime}$. Moreover, since $\operatorname{gcd}(p, c)=$ $\operatorname{gcd}(q, c)=1,\{\mathbf{m}\}$ is of order $c$ and, therefore, generates $\mathcal{G}_{\alpha}^{\prime}$.

For the third part of the proposition, remark that $\mathcal{G}_{\alpha}$ and $\mathbb{Z} \mathbf{m}+\mathbb{Z} \mathbf{m}^{\prime}$ both are invariant by integer translation, thus each of these groups is defined by its intersection with the cell $\left[-\frac{1}{2}, \frac{1}{2}\left[^{2}\right.\right.$. Moreover, $\mathcal{G}_{\alpha}$ contains $\mathbb{Z} \mathbf{m}+\mathbb{Z} \mathbf{m}^{\prime}$ and $\mathcal{G}_{\alpha} \cap\left[-\frac{1}{2}, \frac{1}{2}\left[^{2}\right.\right.$ (which is $\mathcal{G}_{\alpha}^{\prime}$ ) contains exactly $c$ elements.

\footnotetext{
${ }^{1}$ see for example [10] or [3]
} 

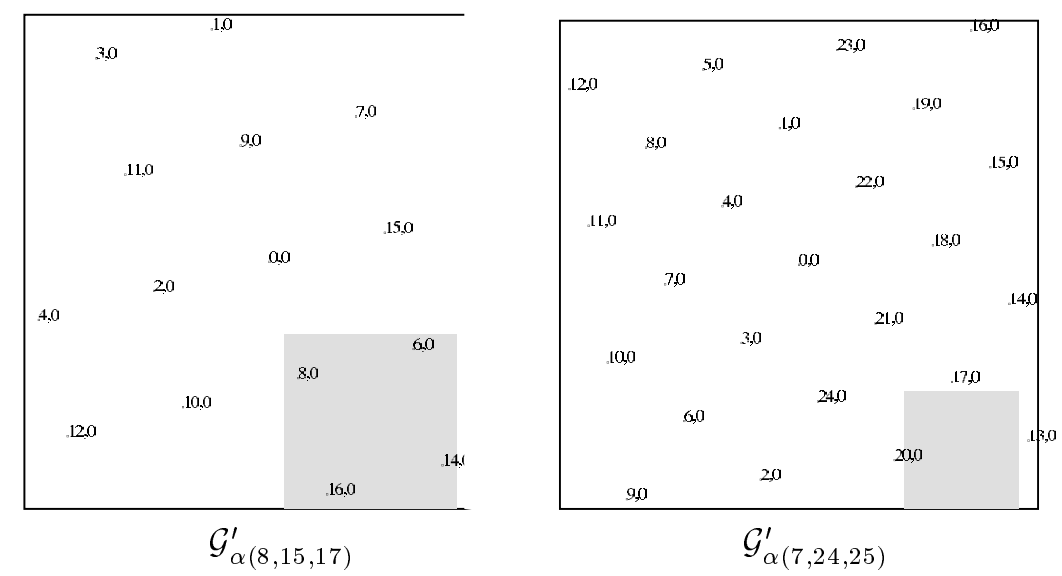

FIG. 3 - On the left, $\mathcal{G}_{\alpha}^{\prime}$ and $F_{0 \downarrow}$ for a non integer pythagorean non integer angle. On the right $\mathcal{G}_{\alpha}^{\prime}$ and $F_{0 \downarrow}$ for a non integer an integer pythagorean angle.

Thus, we only have to prove that $\left(\mathbb{Z} \mathbf{m}+\mathbb{Z} \mathbf{m}^{\prime}\right) \cap\left[-\frac{1}{2}, \frac{1}{2}\left[^{2}\right.\right.$ also contains $c$ elements. In that aim, consider the real plane, seen using the basis $\left(\mathbf{m}, \mathbf{m}^{\prime}\right):$ vectors of $\mathbb{Z} \mathbf{m}+\mathbb{Z} \mathbf{m}^{\prime}$ are seen as the integer vectors. We have $\mathbf{i}=p \mathbf{m}-q \mathbf{m}^{\prime}$, thus the discretization cell of the origin can be seen as a square on which the square lemma can be applied. Thus, the discretization cell of the origin contains $c$ elements of $\mathbb{Z} \mathbf{m}+\mathbb{Z} \mathbf{m}^{\prime}$. This achieves the proof.

\section{$5 \quad$ Results}

\subsection{Proof of the Reciprocal of the Andrès Jacob Theorem}

We now prove the reciprocal of Andrès-Jacob Theorem. The outline of the proof is structured as follows : the main idea of the proof is to show that when we are in the non - integer- pythagorean case it is necessity to have a "hole". This necessity is due to the density of $\mathcal{G}_{\alpha}^{\prime}$ in $\left[-\frac{1}{2}, \frac{1}{2}\left[^{2}\right.\right.$ (or the density of $\mathcal{G}_{\alpha}$ in $\mathbb{Z}^{2}$ ).

Lemma 3 (size lemma) Let $\left[x, x+d\left[\times\left[y, y+d\left[\right.\right.\right.\right.$ be a square of the real plane, with $d \geq \frac{p+q}{c}$. This square has a non-empty intersection with $\mathcal{G}_{\alpha}$.

Proof: We state : $(x, y)=z \mathbf{m}+t \mathbf{m}^{\prime}$. Up to a translation of $\lfloor z\rfloor \mathbf{m}+\lfloor t\rfloor \mathbf{m}^{\prime}$, it can be assumed without loss of generality that $0 \leq z<1$ and $0 \leq t<1$, which gives $-q / c \leq x<p / c$ and $0 \leq y<(p+q) / c$. With this hypothesis we have the case by case analysis below (obtained by cutting the square $\left\{\mathbf{v} \in \mathbb{Z}^{2}, \mathbf{v}=z \mathbf{m}+t \mathbf{m}^{\prime}, 0 \leq z<1,0 \leq t<1\right\}$ by vertical and horizontal lines) :

- for $(x, y)=(0,0)$, the vector $(0,0)$ is in $[x, x+d[\times[y, y+d[$,

- for $-q / c \leq x<(p-q) / c$ and $0<y<(p+q) / c$, the vector $\mathbf{m}+\mathbf{m}^{\prime}$ is in $[x, x+d[\times[y, y+d[$,

- for $(p-q) / c \leq x<(p+q) / c$ and $0 \leq y \leq q / c$, the vector $\mathbf{m}$ is in $[x, x+d[\times[y, y+d[$,

- for $(p-q) / c \leq x<(p+q) / c$ and $q / c<y<(p+q) / c$, the vector $2 \mathbf{m}+\mathbf{m}^{\prime}$ is in $[x, x+$ $d[\times[y, y+d[$.

Theorem 4 (Reciprocal of Andrès Jacob Theorem) If the angle $\alpha$ is not an integer pythagorean angle then the discretized rotation $\left[r_{\alpha}\right]$ is not bijective.

Proof: We recall that if the angle $\alpha$ does not belongs to the set of pythagorean angles, then, from Corollary 1 of Proposition $3,\left[r_{\alpha}\right]$ is not bijective.

For a second time, assume that the angle $\alpha$ is a pythagorean one. With the conventions and notations used above for pythagorean angles, the length side $f_{0}$ of the side of the square $F_{0 \downarrow}$ is $\cos (\alpha)+\sin (\alpha)-1=\frac{a+b-c}{c}=\frac{2 q(p-q)}{c}$. In order $P_{0}$ to be empty, it is mandatory that side $f_{0}<$ $(p+q) / c$ which gives $2 q(\stackrel{c}{p}-q)<(\stackrel{c}{p}+q)$. 

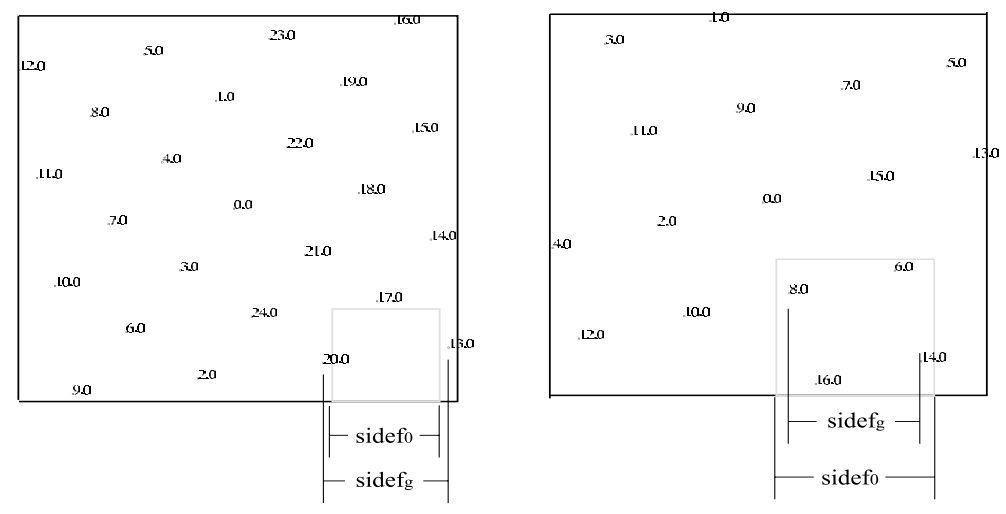

FIG. 4 - side $f_{0}$ and $\frac{p+q}{c}=$ side $f_{g}$ in two samples of Pythagorean angles.

We write $p=q+e$, and thus it is obtained $2 q e<2 q+e$, which may be rewritten as : $(2 q-1) e<2 q$, or $(2 q-1)(e-1)<1$. This is only possible if $e=1$; which is equivalent of saying that $(p, q)$ leads to an integer pythagorean triple. Therefore the discretized rotation cannot bijective for pythagorean non integer angles.

\subsection{Alternate Proof of the Andrès Jacob Theorem}

Theorem 5 (Andrès-Jacob) If the angle of rotation is integer pythagorean, then the discretized euclidean rotation is bijective.

The original proof directly proves the injectivity, with arithmetical arguments. We provide here an alternate one that relies on our framework.

Proof: The main idea of the proof aims to show that there is no point of the group $\mathcal{G}_{\alpha}^{\prime}$ that stands in the "hole frame" $F_{0 \downarrow}$. In order to ensure that the frame is avoided, the position of the points that surround the "hole frame" $F_{0 \downarrow}$ has to be stated precisely.

The frame $F_{0 \downarrow}$ admits the following coordinates :

$$
\begin{aligned}
F_{0 \downarrow} & =\left[\left(F_{0 \downarrow}\right)_{L},\left(F_{0 \downarrow}\right)_{R}\left[\times\left[\left(F_{0 \downarrow}\right)_{D},\left(F_{0 \downarrow}\right)_{U}[\right.\right.\right. \\
& =\left[\frac{1}{2}-\sin (\alpha),-\frac{1}{2}+\cos (\alpha)\left[\times\left[-\frac{1}{2},-\frac{3}{2}+\cos (\alpha)+\sin (\alpha)\right]\right.\right. \\
& =\left[\frac{c-2 b}{2 c}, \frac{2 a-c}{2 c}\left[\times\left[\frac{-c}{2 c}, \frac{2 a+2 b-3 c}{2 c}[\right.\right.\right. \\
& =\left[\frac{p^{2}-3 q^{2}}{2 c}, \frac{p^{2}-3 q^{2}}{2 c}\left[\times\left[\frac{-c}{2 c}, \frac{2 a+2 b-3 c}{2 c}[\right.\right.\right. \\
& =\left[\frac{-2 k^{2}-2 k+1}{2 c}, \frac{-2 k^{2}+2 k+1}{2 c}\left[\times\left[\frac{-2 k^{2}-2 k-1}{2 c}, \frac{-2 k^{2}+2 k-1}{2 c}\right]\right.\right.
\end{aligned}
$$

We have assumed that $\alpha$ is the $k$-th integer pythagorean angle (i. e. $p=k+1$ and $q=k$ ); this yields to $a=p^{2}-q^{2}=2 k+1, b=2 p q=2 k(k+1)$ and $c=p^{2}+q^{2}=2 k^{2}+2 k+1$.

With this hypothesis, we also have $: \mathbf{m}=\left(\frac{k+1}{c}, \frac{k}{c}\right)$ and $\mathbf{m}^{\prime}=\left(\frac{-k}{c}, \frac{k+1}{c}\right)$. Consider the four following vectors :

$$
\begin{aligned}
& -\mathbf{a}=\left(\frac{-2 k^{2}-2 k}{2 c}, \frac{-2 k^{2}}{2 c}\right)=\left(\frac{-k^{2}-k}{c}, \frac{-k^{2}}{c}\right), \\
& -\mathbf{b}=\mathbf{a}+\mathbf{m}=\left(\frac{-2 k^{2}+2}{2 c}, \frac{-2 k^{2}+2 k}{2 c}\right)=\left(\frac{-k^{2}+1}{c}, \frac{-k^{2}+k}{c}\right), \\
& -\mathbf{c}=\mathbf{a}+\mathbf{m}-\mathbf{m}^{\prime}=\left(\frac{-2 k^{2}+2 k+2}{2 c}, \frac{-2 k^{2}-2}{2 c}\right)=\left(\frac{-k^{2}+k+1}{c}, \frac{-k^{2}-1}{c}\right), \\
& -\mathbf{d}=\mathbf{a}-\mathbf{m}^{\prime}=\left(\frac{-2 k^{2}}{2 c}, \frac{-2 k^{2}-2 k-2}{2 c}\right)=\left(\frac{-k^{2}}{c}, \frac{-k^{2}-k-1}{c}\right)
\end{aligned}
$$




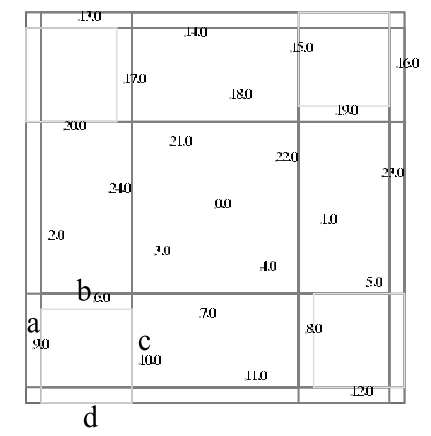

FIG. 5 - The position of the points $\mathbf{a}, \mathbf{b}, \mathbf{c}$ and $\mathbf{d}$ relatively to the hole frame

According to these definitions, we see that these points surround $F_{0 \downarrow}: \mathbf{a}_{x}\left\langle\left(F_{0 \downarrow}^{\prime}\right)_{L}, \mathbf{b}_{y}\right\rangle$ $\left(F_{0 \downarrow}^{\prime}\right)_{U}, \mathbf{c}_{x}>\left(F_{0 \downarrow}^{\prime}\right)_{R}$ and $\mathbf{d}_{y}<\left(F_{0 \downarrow}^{\prime}\right)_{D}$. Therefore we conclude that $F_{0 \downarrow}$ is contained in the square ] $\mathbf{a}_{x}, \mathbf{c}_{x}[\times] \mathbf{d}_{y}, \mathbf{b}_{y}[$.

On the other hand, a belongs to $\mathcal{G}_{\alpha}$, from the membership criterion :

$$
\begin{aligned}
\operatorname{det}\left(\begin{array}{cc}
-k^{2}-k & a \\
-k^{2} & b
\end{array}\right) & =\operatorname{det}\left(\begin{array}{cc}
-k^{2}-k & 2 k+1 \\
-k^{2} & 2 k(k+1)
\end{array}\right) \\
& =-2 k^{4}-2 k^{3}-k^{2} \\
& =-k^{2}\left(2 k^{2}+2 k+1\right) \\
& =-k^{2} c \\
& \equiv 0[c]
\end{aligned}
$$

Since a belongs to the $\mathcal{G}_{\alpha}$, this implies that $\mathbf{b}, \mathbf{c}$ and $\mathbf{d}$ also do. Thus $] \mathbf{a}_{x}, \mathbf{c}_{x}[\times] \mathbf{d}_{y}, \mathbf{b}_{y}[$ does not meet $\mathcal{G}_{\alpha}$, since each element of this square is at distance lower than $\|\mathbf{m}\|=\frac{\sqrt{c}}{c}$ to one of the vectors $\mathbf{a}, \mathbf{b}, \mathbf{c}$, or $\mathbf{d}$. Therefore, the set $F_{0 \downarrow} \cap \mathcal{G}_{\alpha}$ is empty, thus $F_{0 \downarrow} \cap \mathcal{G}_{\alpha}^{\prime}$ is empty, which gives the result from theorem $1 .{ }^{2}$

\section{Conclusion}

The choice of the rounding operator as the discretization function matters for the result : For instance, if we would have taken the floor function as discretization operator, there is no hope to have a bijective rotation : since as long as $\alpha>0,\left\lfloor r_{\alpha}(0,0)\right\rfloor=\left\lfloor r_{\alpha}(\mathbf{i})\right\rfloor$.

While there exists a span of possible discretization functions in between the floor and the rounding, it is the rounding discretization that brings the best point-by-point discretization. The arguments used to prove non-bijectivity hold for any discretization : the only possible bijective rotations are those associated to integer pythagorean angles.

The characterization of angles such that the discretized rotations is bijective, which are somehow the angles for which the discretized rotations has good properties : everything is finite; therefore they are suitable for computations. It has lead to scientific knowledge on the way rotations work : we have got a complete description of $G_{\alpha}^{\prime}$ for pythagorean angles. However, there are others classes of angles, such as the hinge angles [8] that should be studied, and these angles seem to be a source of wonderfully challenging problems.

\footnotetext{
${ }^{2}$ At the following URL, http ://perso.ens-lyon.fr/bertrand.nouvel/work/proofbij.mupad, a mupad session that contains all the proof of our work has been saved, and the interested reader may consult it.
} 


\section{Références}

[1] Eric Andrès. Discrete Circles, and Discrete Rotations. PhD thesis, Université Louis Pasteur, 1992.

[2] Eric Andres. Habilitation à diriger des recherches : Modélisation analytique discrète d'objets géometriques, 2000.

[3] Jean-Marc Chassery and Annick Montanvert Géométrie discrète en analyse d'images Hermes mai, 1991.

[4] G.H. Hardy and E.M. Wright An Introduction to the Theory of Numbers Oxford University Press, London, 1979.

[5] Marie-André Jacob and Eric Andrès. On Discrete Rotations. In Discrete Geometry for Computer Imagery, 1995. LLAIC editions (Univ. Clermont-Ferrand 1).

[6] Bertrand Nouvel. Action des rotations sur le voisinage dans le plan discret. Master's thesis, ENS-Lyon, 2002.

[7] Bertrand Nouvel and Eric Rémila. On Colorations Induced by Discrete Rotations. In Discrete Geometry for Computer Imagery, 2003. Lecture Notes in Computer Science, no 2886.

[8] Bertrand Nouvel. An Incremental Transitive Algorithm for the Discretized Rotations. Submitted to Discrete Geometry for Computer Imagery, 2005.

[9] Jean Pierre Réveillès. Géométrie disrète, Calcul en nombre entiers, et Algorithmique. PhD thesis, Université Louis Pasteur, 1991.

[10] Klaus Voss. Discrete Images, Objects and Functions in $\mathbb{Z}^{n}$ Springer, Berlin, 1993 